\title{
Ambivalent dopamine
}

\section{Hyojung Seo*}

Department of Neurobiology, Yale University School of Medicine, New Haven, CT, USA

${ }^{*}$ Correspondence: hyojung.seo@yale.edu

\section{A commentary on}

Activation in the VTA and nucleus accumbens increases in anticipation of both gains and losses

by R. McKell Carter, JeffJ. MacInnes, Scott A. Huettel and R. Alison Adcock. Front. Behav. Neurosci. (2009), 3:21

Many of animal behaviors can be considered as the end product of the motivation to maximize benefits and minimize costs to the animal. Through intensive investigation based on psychopharmacological manipulations, dopamine in the mammalian nucleus accumbens (NAcc) has been implicated to play a key role in multiple aspects of motivational control of behaviors. However, the exact mechanism by which dopamine produces its behavioral effect is still debated (Wise, 2004; Salamone et al., 2005; Berridge, 2007). A central issue in this debate concerns the role of NAcc dopamine along the two main axes of motivational control, salience and valence. Valence view supports the role of dopamine in mediating appetitive value of the primary reward or conditioned stimuli as well as in reinforcement learning. On the contrary, salience view emphasizes its role in evoking conditioned motivation or "wanting" in the presence of stimuli predicting primary reward, which can engage animals in exerting efforts to obtain predicted reward.

Neurophysiological investigations on the activity of dopamine neurons and their actions in the striatum can provide important clues. For example, activity of dopamine neurons or their reflection in the striatum that are similar for appetitive (e.g., juice or monetary gain) and aversive (e.g., air puff or monetary loss) events would suggest a common motivational component or salience of different stimuli that might have opposite valence. In contrast, modulation of dopamine and striatal activity in the opposite directions by such stimuli would imply a role in encoding motivational valence. A recent study reported both types of modulation in the activity of dopamine neurons in the primate brain and has raised a possibility that distinct populations of dopamine neurons might be involved in representing salience and valence separately (Matsumoto and Hikosaka, 2009).

What is known about the role of the dopamine system in the human brain in coordinating our behaviors in response to aversive events such as monetary losses? Although many previous studies using functional magnetic resonance imaging (fMRI) have characterized the activation in the ventral striatum, including the NAcc, in relation to the evaluation of anticipated and experienced reward and punishment in various contexts, such studies have provided only indirect clues regarding the role of dopamine signals in the striatum, since dopamine neurons constitute only a subset of neural inputs to the striatum.

The fMRI study by Carter et al. (2009) offers an important insight into the role of dopamine signals in human decision making. The authors of this study measured blood oxygen level dependent (BOLD) signals in the NAcc as well as ventral tegmental area (VTA) where dopamine neurons projecting to NAcc are located, while participants performed a monetary incentive reaction time task. During this task, participants were required to respond to visual cues presented on the screen as quickly as possible in order to gain or to avoid losing a particular amount of money. The experiments also included conditions in which gains and losses of money determined by the performance of the participant were donated to a charity, to manipulate the extent of motivational salience of the monetary outcome.

The authors in this study found that BOLD signals in VTA and downstream
NAcc increased above the level observed in the baseline condition ( $\$ 0)$ following the cues indicating potential monetary gains as well as those indicating potential losses. Moreover, the activity enhancement caused by anticipated loss was comparable to the increased activation caused by anticipated gain. These findings challenge the notion that the activation of human dopamine system simply represents either appetitive or aversive value of anticipated outcomes. In contrast, they suggest that human dopamine system might serve heterogeneous functions with regard to dual aspects of motivation (salience and valence) in an intricate way. First, while anticipated gains and losses both increased activation in the VTA and NAcc, the relative strengths of the modulations in BOLD signals associated with gains and losses varied across participants, and this was systematically related to the individual's reward sensitivity. Second, when the money resulting from the participant's performance was donated to a charity, overall activation level decreased compared to when the money was directed to self. In addition, when the money was donated to a charity, the valence of anticipated outcome did not modulate activation in the way reflecting individual's subjective sensitivity to reward.

This work by Carter and colleagues provides new evidence that human dopamine system might be involved in both valencedependent and valence-independent aspect of motivation. It is possible that these two distinct but related functions might be carried out by separate pathways within the dopamine system. Matsumoto and Hikosaka (2009) had previously reported separate population of dopamine neurons, one being suppressed and the other being excited by the anticipation of aversive event. These separate groups of neurons are likely to receive inputs from distinct brain regions as well (Matsumoto and Hikosaka, 2007). Carter and colleague's findings also suggest that the 
relative efficacy of the valence-dependent pathway might vary across individuals and can be flexibly modulated across different behavioral contexts. However, the precise nature of neural circuits implementing multiple motivational functions through potentially heterogeneous dopamine system still remains unknown. For example, what are the distinct sources of inputs to heterogeneous populations of dopamine neurons, and how do diverse behavioral contexts dynamically change the balance between these multiple pathways? How do these different types of dopamine signals diverge or converge in the downstream target areas, including the NAcc? All these questions await further investigations.

\section{REFERENCES}

Carter, R., MacInnes, J., Huettel, S. A., and Adcock, R. (2009). Activation in the VTA and nucleus accumbens increases in anticipation of both gains and losses. Front. Behav. Neurosci. 3, 21.

Berridge, K. C. (2007). The debate over dopamine's role in reward: the case for incentive salience. Psychopharmacology 191, 391431.

Matsumoto, M., and Hikosaka, O. (2007). Lateral habenula as a source of negative reward signals in dopamine neurons. Nature 447, 1111-1115.

Matsumoto, M., and Hikosaka, O. (2009). Two types of dopamine neuron distinctly convey positive and negative motivational signals. Nature 459, 837-841.
Salamone, J. D., Correa, M., Mingote, S. M., and Weber, S. M. (2005). Beyond the reward hypothesis: alternative functions of nucleus accumbens dopamine. Curr. Opin. Pharmacol. 5, 34-41.

Wise, R. A. (2004). Dopamine, learning and motivation. Nat. Rev. Neurosci. 5, 483-494.

Received: 08 March 2010; paper pending published: 27 May 2010; accepted: 27 May 2010; published online: 15 September 2010.

Citation: Seo H (2010) Ambivalent dopamine. Front. Neurosci. 4:38. doi: 10.3389/fnins.2010.00038

Copyright (c) 2010 Seo. This is an open-access article subject to an exclusive license agreement between the authors and the Frontiers Research Foundation, which permits unrestricted use, distribution, and reproduction in any medium, provided the original authors and source are credited. 